\title{
Numerical Consideration of Chen-Lee-Liu Equation through Modification Method for Various Types of Solitons
}

\author{
A. S. H. F. Mohammed*, O. H. Bakodah \\ Department of Mathematics, Faculty of Science, University of Jeddah, Jeddah, KSA \\ Email: *ashmohamad@uj.edu.sa
}

How to cite this paper: Mohammed, A.S.H.F. and Bakodah, O.H. (2020) Numerical Consideration of Chen-Lee-Liu Equation through Modification Method for Various Types of Solitons. American Journal of Computational Mathematics, 10, 398-409. https://doi.org/10.4236/ajcm.2020.103021

Received: June 16, 2020

Accepted: July 21, 2020

Published: July 24, 2020

Copyright $\odot 2020$ by author(s) and Scientific Research Publishing Inc. This work is licensed under the Creative Commons Attribution International License (CC BY 4.0).

http://creativecommons.org/licenses/by/4.0/

\begin{abstract}
The purpose of the current study is to assess the effectiveness and exactness of the new Modification of the Adomian Decomposition (MAD) method in providing fast converging numerical solutions for the Chen-Lee-Liu (CLL) equation. In addition, we are able to simulate the scheme and provide a comparative analysis with the help of some exact soliton solutions in optical fibers. Finally, the MAD method uncovered that the strategy is proven to be reliable due to the elevated level of accuracy and less computational advances, as demonstrated by a series of tables and figures.
\end{abstract}

\section{Keywords}

Chen-Lee-Liu Equation, Solitary Wave, the New Modification of the Adomian Decomposition

\section{Introduction}

In 2001, Wazwaz and El-Sayed proposed another powerful Modification of the Adomian Decomposition (MAD) method [1]. In this modification, the function $f(x)$ that normally emanates from the given initial condition and source function (when prescribed) is decomposed into infinite components via the application of the Taylor's series. In fact, this is contrary to the reliable modified technique of Adomian Decomposition Method (ADM) which decomposes the function $f(x)$ into only two components $f_{1}(x)$ and $f_{2}(x)$ [2]. This modified method of decomposition has been shown to be numerically efficient in several mathematical models that arise in many applications of science and engineering [1] [2] [3] [4]. However, we aim in this paper to examine the type-II member of 
the class of Derivative Nonlinear Schrodinger Equations (DNSE) called the Chen-Lee-Liu (CLL) equation [5] using the MAD method. The equation was introduced in 1979 as an integrable model with a variety of applications, including ultrashort pulse propagation and modeling optical and photonic crystal fibers among others. More, the dimensionless form of the model for pulse propagation in a single-mode optical fiber is given as follows [5]:

$$
i q_{t}+a q_{x x}+b|q|^{2} q_{x}=0
$$

where $q=q(x, t)$ is a complex-valued function denoting the wave propagation profile in space $x$ and time $t$, variables; $a$ and $b$ are real constants. Physically, the parameter $a$ is group-velocity dispersion, while the parameter $b$ denotes the self-steepening phenomena in optical fiber sense. Also, a Regular CLL (RCLL) equation is obtained from Equation (1) by setting $a=b=1$.

In addition, there have been several analytical considerations regarding the existence of valid exact optical soliton solutions for the CLL equation via various analytical methods [6] [7] [8]. Some of these solutions and their respective initial conditions are considered as follows:

1) Bright solitons

a) The first exact bright soliton solution of Equation (1) is given [6] as follows:

$$
q(x, t)=\frac{A \operatorname{sech}[\eta(x-v t)]}{\sqrt{1+B \operatorname{sech}^{2}[\eta(x-v t)]}} \mathrm{e}^{\mathrm{i}[-k x+\omega t+\theta(x-v t)]}
$$

with the corresponding initial condition

$$
q(x, 0)=\frac{A \operatorname{sech}(\eta x)}{\sqrt{1+B \operatorname{sech}^{2}(\eta x)}} \mathrm{e}^{-i k x}
$$

where

$$
\begin{gathered}
A=\sqrt{\frac{-2 \delta(2 B+1)}{\sigma}}, \eta=\sqrt{-\delta} \text { and } 2 B+1= \pm\left(1-\frac{16 \gamma \delta}{3 \sigma^{2}}\right)^{-\frac{1}{2}} \\
\forall \delta<0, \sigma>0 \text { and } \gamma<\left|\frac{3 \sigma^{2}}{16 \delta}\right|
\end{gathered}
$$

b) The second exact bright soliton solution of Equation (1) is given [6] as follows:

$$
q(x, t)=\frac{P}{\sqrt{1+r \cosh [\rho(x-v t)]+\lambda \sinh [\rho(x-v t)]}} \mathrm{e}^{i[-k x+\omega t+\theta(x-v t)]}
$$

with the corresponding initial condition

$$
q(x, 0)=\frac{P}{\sqrt{1+r \cosh (\rho x)+\lambda \sinh (\rho x)}} \mathrm{e}^{-i k x}
$$

where

$$
P^{2}=-\frac{4 \delta}{\sigma}, \rho^{2}=-4 \delta, r^{2}=1+\lambda^{2}-\frac{16 \gamma \delta}{3 \sigma^{2}}
$$




$$
\forall \delta<0, \quad \sigma>0 \text { and } \gamma<\left|\frac{3 \sigma^{2}\left(1+\lambda^{2}\right)}{16 \delta}\right|
$$

\section{2) Dark solitons}

a) The exact dark soliton solution of Equation (1) is given in [7] as follows:

$$
q(x, t)=p \sqrt{1-\operatorname{sech}[\mu(x-v t)]} \mathrm{e}^{i[-k x+\omega t+\theta(x-v t)]}
$$

with the corresponding initial condition

$$
q(x, 0)=p \sqrt{1-\operatorname{sech}(\mu x)} \mathrm{e}^{-i k x}
$$

where

$$
p=\sqrt{-\frac{8 \delta}{5 \sigma}}, \mu=\sqrt{\frac{4 \delta}{5}}, \gamma=\frac{15 \sigma^{2}}{64 \delta}
$$

$\forall \delta>0$ and $\sigma<0$.

b) The exact gray soliton solution of Equation (1) is given in [7] as follows:

$$
q(x, t)=\frac{\lambda \cosh [\mu(x-v t)]}{\sqrt{\epsilon+\cosh ^{2}[\mu(x-v t)]}} \mathrm{e}^{i[-k x+\omega t+\theta(x-v t)]}
$$

with the corresponding initial condition

$$
q(x, 0)=\frac{\lambda \cosh (\mu x)}{\sqrt{\epsilon+\cosh ^{2}(\mu x)}} \mathrm{e}^{-i k x}
$$

where

$$
\delta=-\frac{\mu^{2}(\epsilon+3)}{\epsilon}, \sigma=\frac{2 \mu^{2}(2 \epsilon+3)}{\lambda^{2} \epsilon}, \gamma=-\frac{3 \mu^{2}(\epsilon+1)}{\lambda^{4} \epsilon}
$$

$\forall \delta<0, \sigma>0 ; \lambda, \epsilon, v, \omega, k$ and $\mu$ are arbitrary constants.

\section{3) Singular solitons}

a) The first exact singular soliton solution of Equation (1) is given in [8] as follows:

$$
q(x, t)=p \sqrt{1+\operatorname{coth}[\mu(x-v t)]} \mathrm{e}^{i[-k x+\omega t+\theta(x-v t)]}
$$

with the corresponding initial condition

$$
q(x, 0)=p \sqrt{1+\operatorname{coth}(\mu x)} \mathrm{e}^{-i k x}
$$

where

$$
\begin{gathered}
p=\sqrt{-\frac{2 \delta}{\sigma}}, \mu=\sqrt{-\delta} \\
\forall \delta<0, \sigma>0 \text { and } \gamma=\frac{3 \sigma^{2}}{16 \delta} .
\end{gathered}
$$

b) The second exact singular soliton solution of Equation (1) is given in [8] as follows:

$$
q(x, t)=\frac{p \operatorname{csch}[Z(x-v t)]}{\sqrt{1-R \operatorname{coth}^{2}[Z(x-v t)]}} \mathrm{e}^{i[-k x+\omega t+\theta(x-v t)]}
$$

with the corresponding initial condition 


$$
q(x, 0)=\frac{p \operatorname{csch}(Z x)}{\sqrt{1-R \operatorname{coth}^{2}(Z x)}} \mathrm{e}^{-i k x}
$$

where

$$
p=\sqrt{\frac{2 \delta(1+R)}{\sigma}}, Z=\sqrt{-\delta}, \gamma=\frac{3 \sigma^{2} R}{4 \delta(1+R)^{2}}
$$

$\forall \delta<0, \sigma<0, \gamma>0$ and $R<-1$.

Thus, we shall, therefore, use the aforementioned exact soliton solutions as benchmark solutions for establishing a comparative study with the numerical MAD method. Moreover, the recently constructed bright solitons of CLL equation were numerically confirmed by the ADM [9] and improved ADM [10]. Furthermore, the w-shaped solitons of CLL equation were also validated computationally by coupling of Laplace transform and ADM [11]; see also [4] [12]-[21] for some related Adomian-based methods of decomposition to solve different partial differential equations. The paper is structured as follows: The recursive scheme for the CLL equation is derived in Section 2, using the MAD Method. The results provided by the method are shown and discussed in section 3; while section 4 provides some concluding remarks.

\section{The Describe MAD Method}

This section describes the MAD method for the CLL equation. Firstly, we consider the operator notation by letting $L=\frac{\partial}{\partial t}$ and its corresponding inverse operator $L^{-1}=\int_{0}^{t}(.) \mathrm{d} t$. Employing this inverse $L^{-1}$ on the CLL equation given in Equation (1), we obtain

$$
q=f(x)+a i \int_{0}^{t} q_{x x} \mathrm{~d} t-b \int_{0}^{t} A \mathrm{~d} t
$$

where $f(x)$ emanates from the associated initial condition of the equation; that is, $f(x)=q(x, 0)$, and the nonlinear term $A=|q|^{2} q_{x}$.

The solution $q$ based on the Adomian method is decomposed into a sum of infinite components given by the following series

$$
q=\sum_{n=0}^{\infty} q_{n}
$$

The nonlinear expression $A$ is represented by the sum of infinite Adomian polynomials of the form

$$
A=\sum_{n=0}^{\infty} A_{n}\left(q_{0}, q_{1}, \cdots, q_{n}\right)
$$

where $A_{n}$ are Adomian polynomials that are computed for any form of nonlinearity using the following formula given in compact form as follows

$$
A_{n}=\frac{1}{n !} \frac{\mathrm{d}^{n}}{\mathrm{~d} \lambda^{n}} N\left(\sum_{i=0}^{\infty} \lambda^{i} q_{i}\right)_{\lambda=0}, n \geq 0
$$

Now, using Equation (10) with $A=|q|^{2} q_{x}$, some of the Adomian polynomials $A_{n}$ are calculated by the Equation (11) as follows

$$
A_{0}=q_{0} \overline{q_{0}} q_{0 x}
$$




$$
\begin{aligned}
& A_{1}=q_{1} \overline{q_{0}} q_{0 x}+q_{0} \overline{q_{1}} q_{0 x}+q_{0} \overline{q_{0}} q_{1 x} \\
& A_{2}=q_{2} \overline{q_{0}} q_{0 x}+q_{1} \overline{q_{1}} q_{0 x}+q_{0} \overline{q_{2}} q_{0 x}+q_{1} \overline{q_{0}} q_{1 x}+q_{0} \overline{q_{1}} q_{1 x}+q_{0} \overline{q_{0}} q_{2 x} \\
& A_{3}=q_{3} \overline{q_{0}} q_{0 x}+q_{2} \overline{q_{1}} q_{0 x}+q_{1} \overline{q_{2}} q_{0 x}+q_{0} \overline{q_{3}} q_{0 x}+q_{2} \overline{q_{0}} q_{1 x}+q_{1} \overline{q_{1}} q_{1 x} \\
&+q_{0} \overline{q_{2}} q_{1 x}+q_{1} \overline{q_{0}} q_{2 x}+q_{0} \overline{q_{1}} q_{2 x}+q_{0} \overline{q_{0}} q_{3 x} \\
& A_{4}= q_{4} \overline{q_{0}} q_{0 x}+q_{3} \overline{q_{1}} q_{0 x}+q_{2} \overline{q_{2}} q_{0 x}+q_{1} \overline{q_{3}} q_{0 x}+q_{0} \overline{q_{4}} q_{0 x}+q_{3} \overline{q_{0}} q_{1 x} \\
&+q_{2} \overline{q_{1}} q_{1 x}+q_{1} \overline{q_{2}} q_{1 x}+q_{0} \overline{q_{3}} q_{1 x}+q_{2} \overline{q_{0}} q_{2 x}+q_{1} \overline{q_{1}} q_{2 x}+q_{0} \overline{q_{2}} q_{2 x} \\
&+q_{1} \overline{q_{0}} q_{3 x}+q_{0} \overline{q_{1}} q_{3 x}+q_{0} \overline{q_{0}} q_{4 x}
\end{aligned}
$$

The new modification [1] suggests that the function $f(x)$ be expressed in Taylor series

$$
f(x)=\sum_{n=0}^{\infty} f_{n}(x)
$$

Consequently, putting Equations (9)-(12) in Equation (8), we get the general solution recursively as follows

$$
\left\{\begin{array}{l}
q_{0}=f_{0}(x), \\
q_{k+1}=f_{k+1}(x)+a i \int_{0}^{t} q_{k x x} \mathrm{~d} t-b \int_{0}^{t} A_{k} \mathrm{~d} t, k \geq 0 .
\end{array}\right.
$$

Therefore, the aiming general recursive scheme by the MAD method is determined in Equation (13); this scheme will be simulated alongside the exact soliton solutions given in Section lin the next section.

\section{Results and Discussions}

This section presents the obtained numerical results using the said method and carries out some comparative analysis. Considering bright, dark, gray and singular soliton solutions, we are able to numerically simulate the derived recursive scheme with the help of the Maple software and present the corresponding absolute error analysis in Tables 1-6 and their respective graphical representations in Figures 1-12. Looking at the minimal error discrepancies revealed, it is noted that the MAD approach performs effectively in respect of the benchmark solutions under consideration; this also is in conformity with most related numerical literature that the MAD method performs greatly.

Table 1. The absolute error of the MAD method for the first kind of bright solitons of CLL equation when $a=0.03, b=-10, v=10^{-3}, k=10^{-4}$ and $w=10^{-5}$.

\begin{tabular}{cccc}
\hline & & $\left|q_{E}-q_{\text {MAD }}\right|$ & \\
\cline { 2 - 4 } & $t=0.1$ & $t=0.3$ & $t=0.5$ \\
\hline-3 & $4.941049070 \times 10^{-9}$ & $1.482317224 \times 10^{-8}$ & $2.470522806 \times 10^{-8}$ \\
-2 & $4.941705997 \times 10^{-9}$ & $1.482523539 \times 10^{-8}$ & $2.470871078 \times 10^{-8}$ \\
-1 & $4.942170785 \times 10^{-9}$ & $1.482649605 \times 10^{-8}$ & $2.471083810 \times 10^{-8}$ \\
0 & $4.942302549 \times 10^{-9}$ & $1.482690765 \times 10^{-8}$ & $2.471151274 \times 10^{-8}$ \\
1 & $4.942149932 \times 10^{-9}$ & $1.482646967 \times 10^{-8}$ & $2.471079523 \times 10^{-8}$ \\
2 & $4.941806153 \times 10^{-9}$ & $1.482527998 \times 10^{-8}$ & $2.470885735 \times 10^{-8}$ \\
3 & $4.941073165 \times 10^{-9}$ & $1.482319533 \times 10^{-8}$ & $2.470533641 \times 10^{-8}$ \\
\hline
\end{tabular}


Table 2. The absolute error of the MAD method for the second kind of bright solitons of CLL equation when $a=\alpha=10^{-2}, b=-10, v=10^{-3}, k=10^{-5}$ and $w=10^{-4}$.

\begin{tabular}{cccc}
\hline & \multicolumn{3}{c}{$\left|q_{E}-q_{\text {MAD }}\right|$} \\
\cline { 2 - 4 } & $t=0.1$ & $t=0.3$ & $t=0.5$ \\
\hline-3 & $3.490142858 \times 10^{-8}$ & $1.047034922 \times 10^{-7}$ & $1.745048753 \times 10^{-7}$ \\
-2 & $3.760986543 \times 10^{-8}$ & $1.128292002 \times 10^{-7}$ & $1.880476928 \times 10^{-7}$ \\
-1 & $3.939351251 \times 10^{-8}$ & $1.181805534 \times 10^{-7}$ & $1.969668890 \times 10^{-7}$ \\
0 & $3.999454342 \times 10^{-8}$ & $1.199836437 \times 10^{-7}$ & $1.999727533 \times 10^{-7}$ \\
1 & $3.932113861 \times 10^{-8}$ & $1.179636145 \times 10^{-7}$ & $1.966065171 \times 10^{-7}$ \\
2 & $3.747566437 \times 10^{-8}$ & $1.124275103 \times 10^{-7}$ & $1.873800097 \times 10^{-7}$ \\
3 & $3.472394786 \times 10^{-8}$ & $1.041723244 \times 10^{-7}$ & $1.736215630 \times 10^{-7}$ \\
\hline
\end{tabular}

Table 3. The absolute error of the MAD method for the dark soliton of CLL equation when $a=v=10^{-4}, b=10$ and $k=w=10^{-5}$.

\begin{tabular}{cccc}
\hline & \multicolumn{3}{c}{$\left|q_{E}-q_{\text {MAD }}\right|$} \\
\cline { 2 - 4 } & $t=0.1$ & $t=0.3$ & $t=0.5$ \\
\hline-3 & $3.408520537 \times 10^{-9}$ & $1.022557878 \times 10^{-8}$ & $1.704192591 \times 10^{-8}$ \\
-2 & $4.198070193 \times 10^{-9}$ & $1.259422605 \times 10^{-8}$ & $2.099049057 \times 10^{-8}$ \\
-1 & $5.006381143 \times 10^{-9}$ & $1.501953589 \times 10^{-8}$ & $2.503260278 \times 10^{-8}$ \\
1 & $5.006378921 \times 10^{-9}$ & $1.501942179 \times 10^{-8}$ & $2.503255387 \times 10^{-8}$ \\
2 & $4.198065064 \times 10^{-9}$ & $1.259391626 \times 10^{-8}$ & $2.099018573 \times 10^{-8}$ \\
3 & $3.407046316 \times 10^{-9}$ & $1.022405804 \times 10^{-8}$ & $1.704031246 \times 10^{-8}$ \\
\hline
\end{tabular}

Table 4. The absolute error of the MAD method for the gray soliton of CLL equation when $a=1, b=1, \mu=0.1, v=k=w=\epsilon=0.001$ and $\lambda=10^{-6}$.

\begin{tabular}{cccc}
\hline & \multicolumn{3}{c}{$\left|q_{E}-q_{\text {MAD }}\right|$} \\
\cline { 2 - 4 }$x$ & $t=0.1$ & $t=0.3$ & $t=0.5$ \\
\hline-3 & $9.926833579 \times 10^{-10}$ & $2.978140609 \times 10^{-9}$ & $4.963598661 \times 10^{-9}$ \\
-2 & $9.910304091 \times 10^{-10}$ & $2.973118609 \times 10^{-9}$ & $4.955207967 \times 10^{-9}$ \\
-1 & $9.899120579 \times 10^{-10}$ & $2.969740106 \times 10^{-9}$ & $4.949569139 \times 10^{-9}$ \\
0 & $9.895153635 \times 10^{-10}$ & $2.968546259 \times 10^{-9}$ & $4.947577640 \times 10^{-9}$ \\
1 & $9.899152583 \times 10^{-10}$ & $2.969742165 \times 10^{-9}$ & $4.949568936 \times 10^{-9}$ \\
2 & $9.910568611 \times 10^{-10}$ & $2.973143360 \times 10^{-9}$ & $4.955229306 \times 10^{-9}$ \\
3 & $9.927731452 \times 10^{-10}$ & $2.978228977 \times 10^{-9}$ & $4.963684231 \times 10^{-9}$ \\
\hline
\end{tabular}

Table 5. The absolute error of the MAD method for the first kind of singular solitons of CLL equation when $a=v=k=w=10^{-6}$ and $b=-10$.

\begin{tabular}{cccc}
\hline & \multicolumn{3}{c}{$\left|q_{E}-q_{\text {MAD }}\right|$} \\
\cline { 2 - 4 } & $t=0.1$ & $t=0.3$ & $t=0.5$ \\
\hline-3 & $5.487892336 \times 10^{-12}$ & $1.655185228 \times 10^{-11}$ & $2.757640349 \times 10^{-11}$ \\
\hline
\end{tabular}




\section{Continued}

\begin{tabular}{cccc}
\hline-2 & $1.544323282 \times 10^{-11}$ & $4.533920170 \times 10^{-11}$ & $7.533425774 \times 10^{-11}$ \\
-1 & $8.862920745 \times 10^{-11}$ & $2.653001416 \times 10^{-10}$ & $4.421670840 \times 10^{-10}$ \\
1 & $1.406053708 \times 10^{-10}$ & $4.217227544 \times 10^{-10}$ & $7.028406424 \times 10^{-10}$ \\
2 & $7.635477303 \times 10^{-11}$ & $2.290850955 \times 10^{-10}$ & $3.818155035 \times 10^{-10}$ \\
3 & $7.707209127 \times 10^{-11}$ & $2.311986559 \times 10^{-10}$ & $3.853426395 \times 10^{-10}$ \\
\hline
\end{tabular}

Table 6. The absolute error of the MAD method for the second kind of singular solitons of CLL equation when $a=v=k=0.0001, b=10, w=0.001$ and $R=-6$.

\begin{tabular}{cccc}
\hline & & $\left|q_{E}-q_{\text {MAD }}\right|$ & \\
\cline { 2 - 4 } & \multicolumn{1}{c}{$t=0.1$} & $t=0.3$ & $t=0.5$ \\
\hline-3 & $8.936541858 \times 10^{-11}$ & $2.680042918 \times 10^{-10}$ & $4.465258727 \times 10^{-10}$ \\
-2 & $2.027981400 \times 10^{-9}$ & $6.081814504 \times 10^{-9}$ & $1.013304751 \times 10^{-8}$ \\
-1 & $3.625151370 \times 10^{-8}$ & $1.087261596 \times 10^{-7}$ & $1.811639691 \times 10^{-7}$ \\
1 & $3.626097765 \times 10^{-8}$ & $1.088083658 \times 10^{-7}$ & $1.813956181 \times 10^{-7}$ \\
2 & $2.028661466 \times 10^{-9}$ & $6.087994808 \times 10^{-9}$ & $1.014999902 \times 10^{-8}$ \\
& $8.939474934 \times 10^{-11}$ & $2.682722396 \times 10^{-10}$ & $4.472720510 \times 10^{-10}$ \\
\hline
\end{tabular}
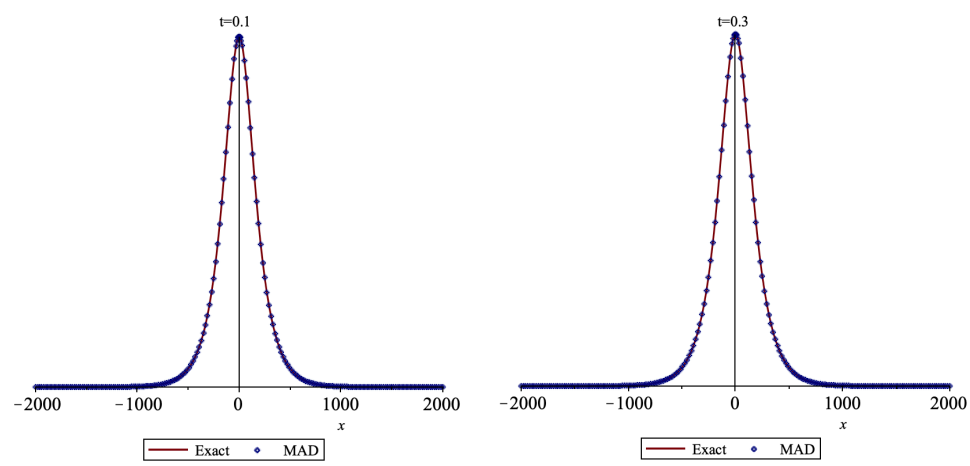

Figure 1. Comparison between the exact and MAD method solutions for the first kind of bright soliton of CLL equation when $t=0.1$ and $t=0.3$.

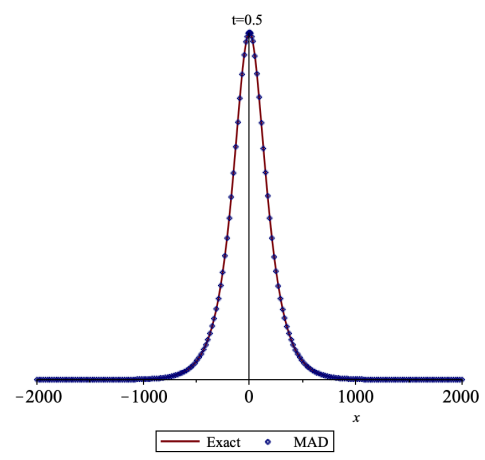

Figure 2. Comparison between the exact and MAD method solutions for the first kind of bright soliton of CLL equation when $t=0.5$. 

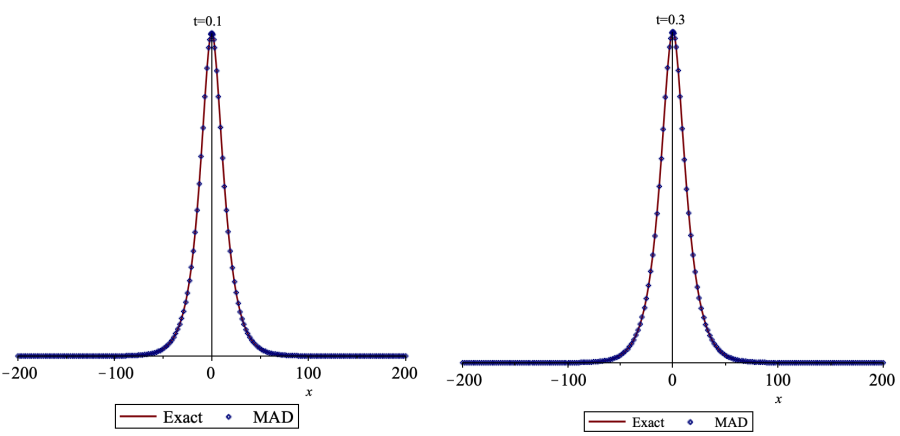

Figure 3. Comparison between the exact and MAD method solutions for the second kind of bright solitons of CLL equation when $t=0.1$ and $t=0.3$.

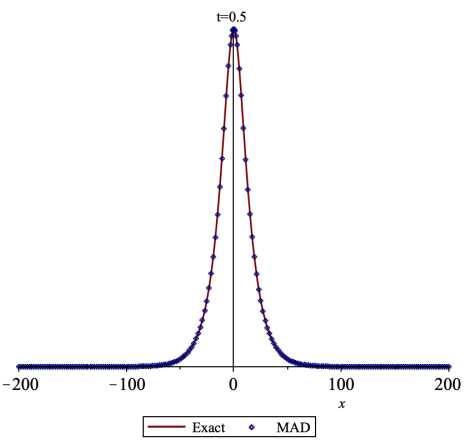

Figure 4. Comparison between the exact and MAD method solutions for the second kind of bright solitons of CLL equation when $t=0.5$.
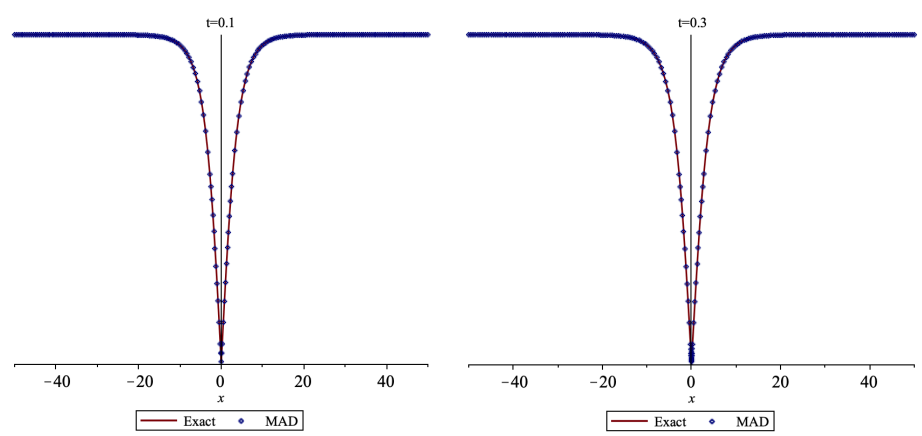

Figure 5. Comparison between the exact and MAD method solutions for the dark soliton of CLL equation when $t=0.1$ and $t=0.3$.

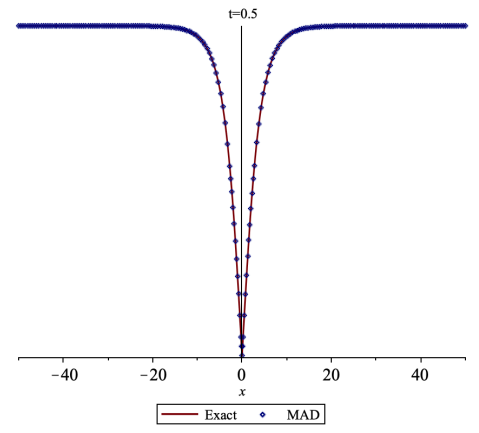

Figure 6. Comparison between the exact and MAD method solutions for the dark soliton of CLL equation when $t=0.5$. 

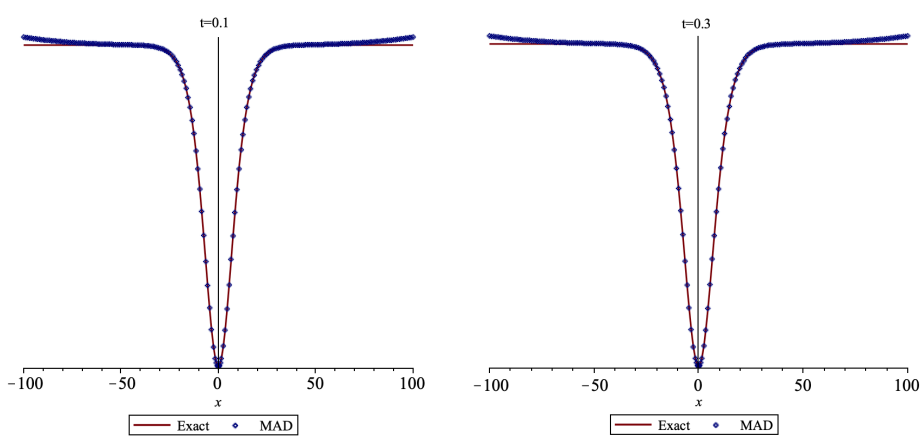

Figure 7. Comparison between the exact and MAD method solutions for the gray soliton of CLL equation when $t=0.1$ and $t=0.3$.

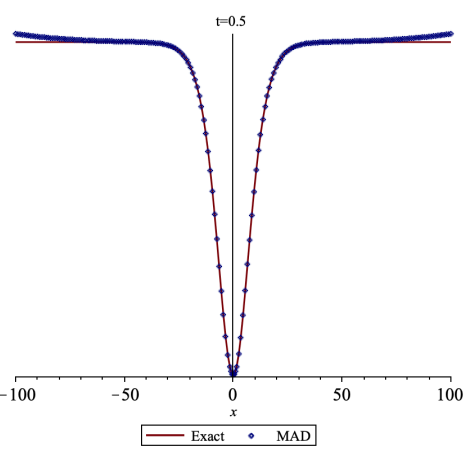

Figure 8. Comparison between the exact and MAD method solutions for the gray soliton of CLL equation when $t=0.5$.
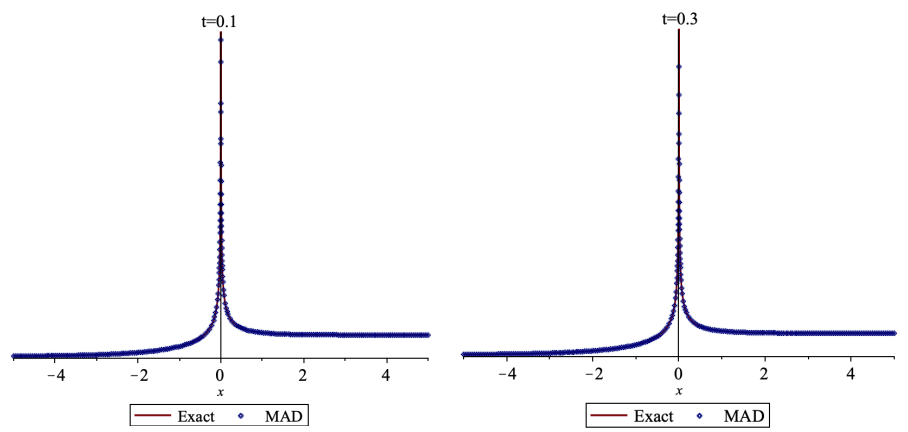

Figure 9. Comparison between the exact and MAD method solutions for the first kind of singular solitons of CLL equation when $t=0.1$ and $t=0.3$.

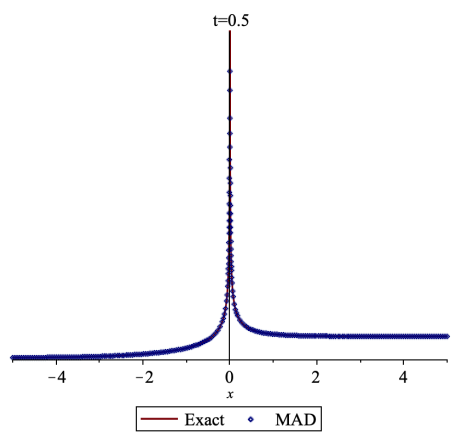

Figure 10. Comparison between the exact and MAD method solutions for the first kind ofsingular solitons of CLL equation when $t=0.5$. 

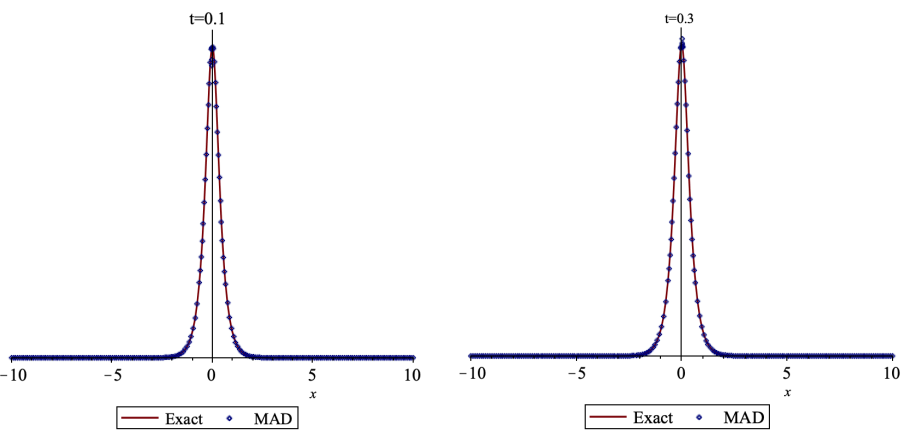

Figure 11. Comparison between the exact and MAD method solutions for the second kind of singular solitons of CLL equation when $t=0.1$ and $t=0.3$.

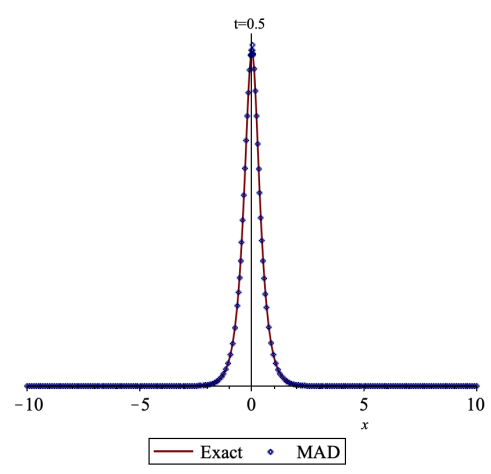

Figure 12. Comparison between the exact and MAD method solutions for the second kind of singular solitons of CLL equation when $t=0.5$.

\section{Conclusion}

In conclusion, a fast converging numerical scheme for the CLL equation was derived using the Wazwaz and El-Sayed MAD method. Recent exact optical solutions in optical fibers including bright, dark, gray and singular solitons have been considered for the numerical simulation and consequently led to the comparative study. The results of this study are showed in Tables 1-6 and illustrated in Figures 1-12, respectively. The comparison of results between the numerical MAD method solutions and the exact soliton solutions for given in Equations (2)-(7) is carried out for different values of $x$ and $t$. In Figures 1-12, we plotted the graphs comparing the two solutions for different time levels including $t=0.1, t=0.3$ and $t=0.5$; while the parameters are arbitrary chosen according to the conditions of each type. The obtained results affirmed the precision and minimal error of the method as demonstrated in the presented tables and figures. Thus, the MAD method is recommended being an amazing approach to solving different kinds of evolution equations with different forms of nonlinearities.

\section{Conflicts of Interest}

The authors declare no conflicts of interest regarding the publication of this paper. 


\section{References}

[1] Wazwaz, A.M. and El-Sayed, S.M. (2001) A New Modification of the Adomian Decomposition Method for Linear and Nonlinear Operators. Applied Mathematics and Computation, 122, 393-405. https://doi.org/10.1016/S0096-3003(00)00060-6

[2] Wazwaz, A.M. (1999) A Reliable Modification of Adomian Decomposition Method. Applied Mathematics Computation, 102, 77-86.

https://doi.org/10.1016/S0096-3003(98)10024-3

[3] Jin, C. and Liu, M. (2005) A New Modification of Adomian Decomposition Method for Solving a Kind of Evolution Equation. Applied Mathematics and Computation, 169, 953-962. https://doi.org/10.1016/j.amc.2004.09.072

[4] Duan, J.S. and Rach, R. (2011) A New Modification of the Adomian Decomposition Method for Solving Boundary Value Problems for Higher Order Nonlinear Differential Equations. Applied Mathematics and Computation, 218, 4090-4118. https://doi.org/10.1016/j.amc.2011.09.037

[5] Chen, H.H., Lee, Y.C. and Liu, C.S. (1979) Integrability of Nonlinear Hamiltonian Systems by Inverse Scattering Method. Physica Scripta, 20, 490. https://doi.org/10.1088/0031-8949/20/3-4/026

[6] Triki, H., Babatin, M.M. and Biswas, A. (2017) Chirped Bright Solitons for Chen-Lee-Liu Equation in Optical Fibers and PCF. Optik-International Journal for Light and Electron Optics, 149, 300-303. https://doi.org/10.1016/j.ijleo.2017.09.031

[7] Triki, H., Hamaizi, Y., Zhou, Q., Biswas, A., Ullah, M.Z., Moshokoa, S.P. and Belic, M. (2018) Chirped Dark and Gray Solitons for Chen-Lee-Liu Equation in Optical Fibers and PCF. Optik-International Journal for Light and Electron Optics, 155, 329-333. https://doi.org/10.1016/j.ijleo.2017.11.038

[8] Triki, H., Hamaizi, Y., Zhou, Q., Biswas, A., Ullah, M.Z., Moshokoa, S.P. and Belic, M. (2018) Chirped Singular Solitons for Chen-Lee-Liu Equation in Optical Fibers and PCF. Optik-International Journal for Light and Electron Optics, 157, 156-160. https://doi.org/10.1016/j.ijleo.2017.11.088

[9] Mohammed, A.S.H.F., Bakodah, H.O., Banaja, M.A., Alshaery, A.A., Zhou, Q., Biswas, A., Belic, M.R., et al. (2019) Bright Optical Solitons of Chen-Lee-Liu Equation with Improved Adomian Decomposition Method. Optik-International Journal for Light and Electron Optics, 181, 964-970. https://doi.org/10.1016/j.ijleo.2018.12.177

[10] Mohammed, A.S.H.F., Bakodah, H.O. and Banaja, M.A. (2019) Approximate Adomian Solutions to the Bright Optical Solitary Waves of the Chen-Lee-Liu Equation. MATTER: International Journal of Science and Technology, 5, 110-120. https://doi.org/10.20319/mijst.2019.53.110120

[11] González-Gaxiola, O. and Biswas, A. (2018) W-Shaped Optical Solitons of Chen-Lee-Liu Equation by Laplace-Adomian Decomposition Method. Optical and Quantum Electronics, 50, 314. https://doi.org/10.1007/s11082-018-1583-0

[12] Al Qarni, A.A., Banaja, M.A., Bakodah, H.O., Alshaery, A.A., Majid, F.B. and Biswas, A. (2016) Optical Solitons in Birefringent Fibers: A Numerical Study. Journal of Computational and Theoretical Nanoscience, 13, 9001-9013. https://doi.org/10.1166/jctn.2016.6077

[13] Bakodah, H.O., Al Qarni, A.A., Banaja, M.A., Zhou, Q., Moshokoa, S.P. and Biswas, A. (2017) Bright and Dark Thirring Optical Solitons with Improved Adomian Decomposition Method. Optik-International Journal for Light and Electron Optics, 130, 1115-1123. https://doi.org/10.1016/j.ijleo.2016.11.123

[14] Banaja, M.A., Al Qarni, A.A., Bakodah, H.O., Zhou, Q., Moshokoa, S.P. and Biswas, 
A. (2017) The Investigate of Optical Solitons in Cascaded System by Improved Adomian Decomposition Scheme. Optik-International Journal for Light and Electron Optics, 130, 1107-1114. https://doi.org/10.1016/j.ijleo.2016.11.125

[15] Biazar, J., Babolian, E., Kember, G., Nouri, A. and Islam, R. (2003) An Alternate Algorithm for Computing Adomian Polynomials in Special Cases. Applied Mathematics and Computation, 138, 523-529.

https://doi.org/10.1016/S0096-3003(02)00174-1

[16] Wazwaz, A.M. (2000) A New Algorithm for Calculating Adomian Polynomials for Nonlinear Operators. Applied Mathematics and Computation, 111, 33-51. https://doi.org/10.1016/S0096-3003(99)00063-6

[17] Nuruddeen, R.I., Muhammad, L., Nass, A.M. and Sulaiman, T.A. (2007) A Review of the Integral Transforms-Based Decomposition Methods and Their Applications in Solving Nonlinear PDEs. Palestine Journal of Mathematics, 7, 262-280.

[18] Rach, R. (2008) A New Definition of the Adomian Polynomials, Kybernetes, 37, 910-955. https://doi.org/10.1108/03684920810884342

[19] Nuruddeen, R.I. (2017) Elzaki Decomposition Method and Its Applications in Solving Linear and Nonlinear Schrodinger Equations. Sohag Journal of Mathematics, 4, 1-5. https://doi.org/10.18576/sjm/040201

[20] Yin, F.K., Wang, Y.H. and Song, J.Q. (2013) Modified Laplace Decomposition Method for Lane-Emden Type Differential. International Journal of Applied Physics and Mathematics, 3, 98-102. https://doi.org/10.7763/IJAPM.2013.V3.184

[21] Babolian, E. and Javadi, S. (2003) Restarted Adomian Method for Algebraic Equations. Applied Mathematics and Computation, 146, 533-541.

https://doi.org/10.1016/S0096-3003(02)00603-3 